\title{
A case of long-term survival after surgical resection for solitary adrenal recurrence of esophageal squamous carcinoma
}

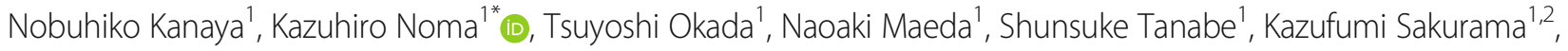 \\ Yasuhiro Shirakawa ${ }^{1}$ and Toshiyoshi Fujiwara ${ }^{1}$
}

\begin{abstract}
Background: Esophageal carcinomas are highly malignant tumors with a high frequency of lymph node and distant organ metastasis. Treatment for recurrent tumors is generally decided on an individual basis. Although multidisciplinary treatments involving chemotherapy, surgical resection, and radiation are performed, the prognosis remains poor. Here, we report a case of prolonged recurrence-free survival (38 months) after esophageal carcinoma surgery and subsequent laparoscopic adrenalectomy for right adrenal metastasis.
\end{abstract}

Case presentation: An 83-year-old man was diagnosed with type 3 esophageal squamous cell carcinoma (T3N1M0, CStage IIIA, UICC-7), spreading from the lower thoracic esophagus to the abdominal esophagus. He underwent thoracoscopic esophagectomy with a two-field lymph node dissection followed by substernal gastric tube reconstruction. The final diagnosis was moderately differentiated squamous cell carcinoma (T3N2M0, fStage IIIB). Adjuvant chemotherapy was not administered because of the advanced age and postoperative condition of the patient. Computed tomography (CT) at 14 months postoperatively showed a mass with a 2-cm diameter at the right adrenal gland. Positron emission tomography (PET)/CT revealed a high fluorodeoxyglucose (FDG) uptake in the mass. It was suspected that the mass was a metastatic lesion secondary to the primary esophageal carcinoma. No metastases to lymph nodes or other distant organs were identified. The patient underwent laparoscopic right adrenalectomy. The histopathological examination revealed moderately differentiated squamous cell carcinoma, suggesting metastasis from the primary esophageal carcinoma. He has survived without recurrence for 38 months since laparoscopic adrenalectomy to remove the right adrenal metastastic mass after the esophageal carcinoma surgery.

Conclusions: We describe a very elderly male who survived laparoadrenalectomy for right adrenal metastasis following esophageal cancer surgery without recurrence for 38 months postoperatively. Therefore, surgical resection might be an option for solitary adrenal recurrence.

Keywords: Esophagectomy, Adrenal metastasis, Esophageal squamous cell carcinoma

\section{Background}

Esophageal carcinomas are highly biologically malignant tumors because the high frequency of lymph node and distant organ metastasis [1]. Common patterns of recurrence are metastasis to the lymph nodes, lungs, liver, bone, brain, and adrenal glands [2,3]. Treatment for recurrent

\footnotetext{
* Correspondence: knoma@md.okayama-u.ac.jp

1Department of Gastroenterological Surgery, Okayama University Graduate

School of Medicine, Dentistry and Pharmaceutical Sciences, 2-5-1

Shikata-cho, Kita-ku, Okayama 700-8558, Japan

Full list of author information is available at the end of the article
}

lesions is generally decided on an individual basis. $\mathrm{Al}$ though multidisciplinary treatments are combined with chemotherapy, surgical resection, and radiation, the prognosis remains poor $[4,5]$. The role of surgical resection for metastasis from esophageal carcinoma has not been clarified, but some reports have recently described the benefit of resection for oligometastasis from esophageal carcinoma $[6,7]$. 


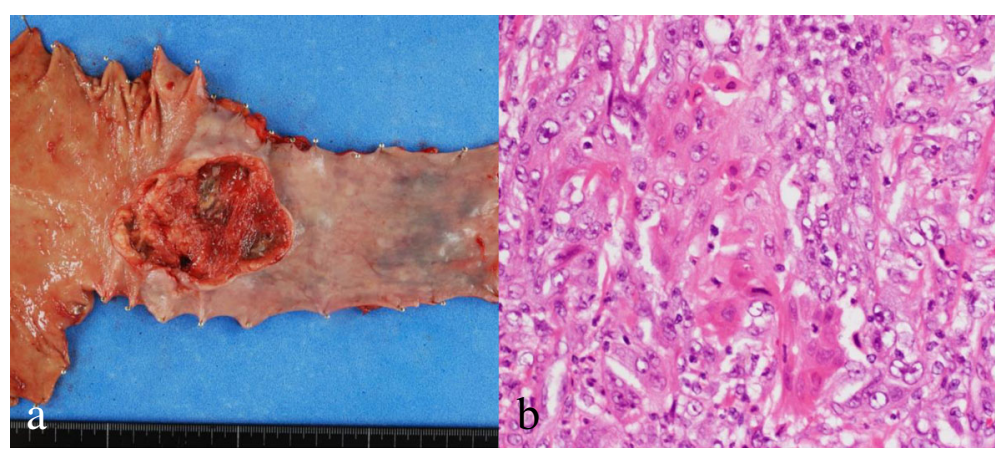

Fig. 1 Resected specimen of esophageal carcinoma. a Gross appearance shows type 3 tumor, approximately 4.5-cm long in LtAe. b Hematoxylin and eosin staining shows moderately differentiated squamous cell carcinoma

Here, we report a case of a 38-month recurrence-free survival after laparoscopic adrenalectomy for right adrenal metastasis after esophageal carcinoma surgery.

\section{Case presentation}

An 83-year-old man was diagnosed with type 3 esophageal squamous cell carcinoma (LtAe, T3N1M0, cStage IIIA, UICC-7) measuring $6 \mathrm{~cm}$ in diameter. Computed tomography (CT) and 8F-fluorodeoxyglucose (FDG) positron emission tomography (PET)/CT showed no metastasis to distant organs, whereas metastatic lymph nodes were present at the lesser curvature of the stomach. The patient underwent thoracoscopic esophagectomy with two-field lymph node dissection, laparoscopic substernal gastric tube reconstruction (Fig. 1). The final diagnosis was moderately differentiated squamous cell carcinoma (T3N2M0, stage IIIB). Three lymph nodes at the lesser curvature of the stomach were diagnosed as containing metastatic squamous cell carcinoma. The patient suffered from diarrhea after the operation. Adjuvant chemotherapy was not administered because of the patient's advancing age and postoperative condition.
After treatment, follow-up was conducted on an outpatient basis once every 3 months. Follow-up included physical examination and laboratory tests, including those for tumor markers. Alternating 6-month periods of upper gastrointestinal endoscopy, contrast-enhanced CT, and FDG-PET/CT were performed. CT at 14 months postoperatively showed a small growing mass, which had enlarged to $2 \mathrm{~cm}$ in diameter in the right adrenal gland (Fig. 2) and PET/CT revealed high FDG uptake at the mass (maximum standardized uptake value (SUV), 7.67). The physical examination at that time showed no abnormalities. Laboratory tests showed that concentrations of squamous cell carcinoma (SCC) antigen and carcinoembryonic antigen (CEA) had increased to $12.3 \mathrm{ng} / \mathrm{ml}$ (normal range, $0-1.5 \mathrm{ng} / \mathrm{ml}$ ) and $18.01 \mathrm{ng} / \mathrm{ml}$ (normal range, $0-5.0 \mathrm{ng} / \mathrm{ml}$ ), although these concentrations had been normal just after the primary operation (Fig. 3). The mass was suspected to be a metastasis from the primary esophageal carcinoma. No metastases to lymph nodes or other distant organs were apparent. The patient was admitted to our hospital for surgery and underwent laparoscopic right adrenalectomy from the left lateral decubitus position. A port for endoscopy was present where

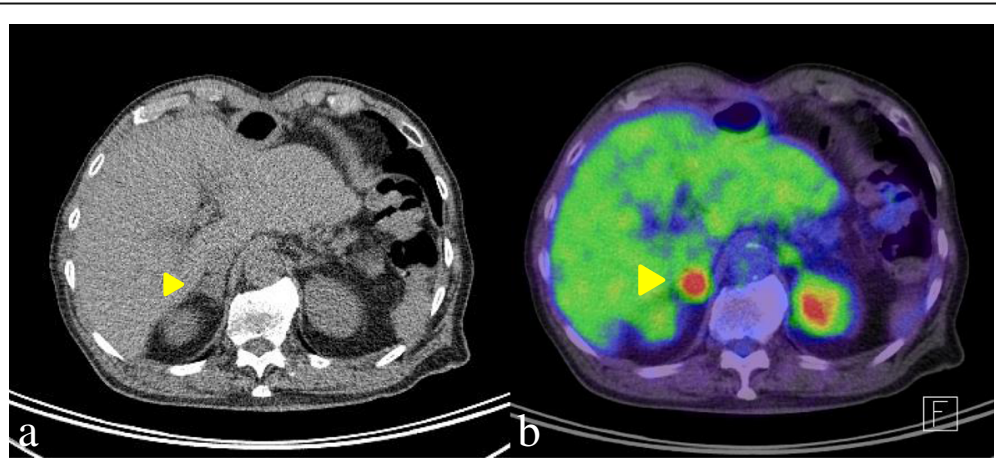

Fig. 2 Images from CT and FDG-PET/CT. a A mass with a 2-cm diameter in the right adrenal gland. b PET/CT shows high accumulation of FDG (standardized uptake value max, 7.67) in the right adrenal mass. Abbreviations: CT computed tomography, PET positron emission tomography, FDG 8F-fluorodeoxyglucose 


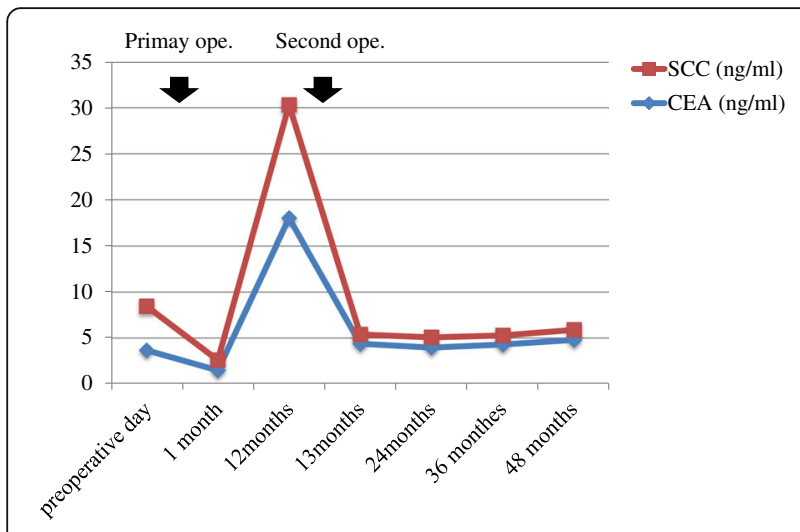

Fig. 3 Clinical changes in concentrations of tumor markers, carcinoembryonic antigen (blue line) and squamous cell carcinoma (red line). Ope operation

the right arcus costalis and external marginal abdominis muscle crossed and the other three ports were along the subcostal lines. If there had been severe intra-abdominal adhesion, then the laparoscopic approach would have been changed to the retroperitoneal approach. Though there was inflammatory adhesion between the inferior vena cava and adipose tissue around the tumor, it was possible to exfoliate them. This second operation ended without any problems because the right adrenal gland was anatomically away from the gastric tube. The operative time was $127 \mathrm{~min}$. The volume of blood loss was $30 \mathrm{ml}$. The histopathological examination revealed moderately differentiated squamous cell carcinoma, strongly suggesting the presence of metastasis from the primary esophageal cancer (Fig. 4). Laboratory tests after this second operation showed decreased concentrations of SCC antigen and CEA to 1 and $4.4 \mathrm{ng} / \mathrm{ml}$, respectively. Thus far, no postoperative recurrences have occurred as of 38 months after adrenalectomy.

\section{Conclusions}

Distant recurrence after curative esophageal carcinoma surgery is one of the most difficult complications to treat. Recently, multidisciplinary therapy has been performed for recurrent esophageal carcinoma. However, the prognosis of the patients with recurrence is very poor, with a reported survival of 4-7 months [8, 9].

The benefit of surgical resection for patients with recurrence remains controversial. Some reports indicated that lymphadenectomy or chemoradiotherapy might improve survival in patients with lymph node recurrence in the neck or mediastinum after curative resection $[4,5]$. Patients with lung metastasis reportedly show relatively good prognosis, with a median survival of 9.8 months [8].Whereas, patients with liver metastasis reportedly exhibit poor prognosis because of the high frequency of multiple metastases [6]. The initial recurrent site could thus contribute to the prognostic heterogeneity of the patients with recurrent esophageal carcinoma.

Most tumors arising in the adrenal gland are benign adenomas [10], and malignant tumors of the adrenal gland are quite rare. The frequency of non-adrenal carcinoma metastases to the adrenal gland is of approximately $0.7-2.5 \%$ [10]. Common carcinomas associated with metastases to the adrenal glands are lung, gastrointestinal, breast, kidney carcinoma, and melanoma. Adrenal metastasis is generally identified on CT, PET, or MRI, during postoperative follow-up $[10,11]$. Therapy for adrenal metastasis is decided on an individual basis depending on the primary carcinoma. The characteristics of adrenal metastasis on imaging studies include an irregular shape, inhomogeneous nature, high vascularity on contrast-enhanced CT, and elevated SUV on PET [11]. In our case, the adrenal mass was found on a follow-up CT and FDG-PET/CT in the absence of other primary lesions. Tumor markers such as SCC and CEA were also elevated. Additionally, FDG uptake was high

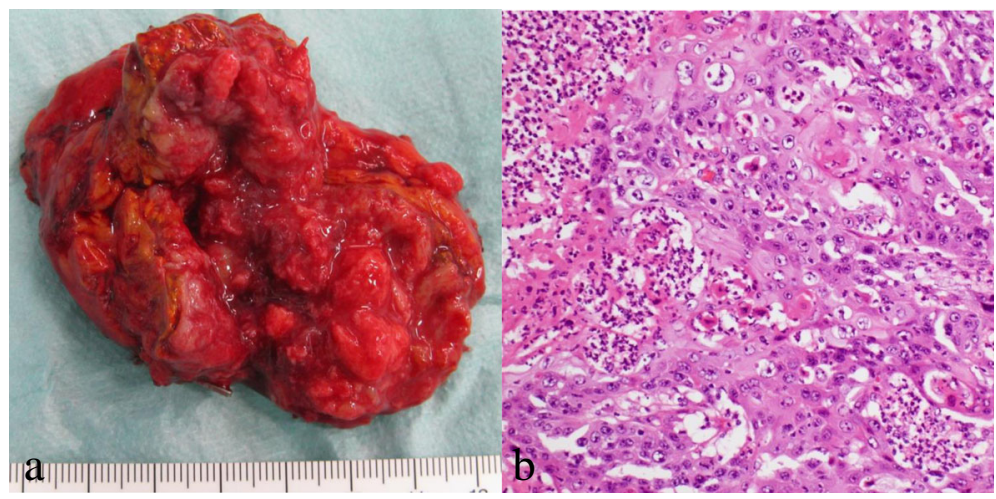

Fig. 4 Resected specimen of the right adrenal mass. a Gross appearance shows a hard, solid mass approximately $2.0 \mathrm{~cm}$ in diameter. b Hematoxylin eosin staining shows moderately differentiated squamous cell carcinoma 
Table 1 Eight patients with surgical resection of adrenal metastasis from esophageal carcinoma

\begin{tabular}{lllllllllll}
\hline Year & Author & Age & Sex & $\begin{array}{l}\text { Location } \\
\text { of the EC }\end{array}$ & Histology fStage $\begin{array}{l}\text { Location } \\
\text { of the AM }\end{array}$ & Size of AM (cm) & Interval from the EC Prognosis & Chemotherapy \\
\hline 1992 & Shimada & 59 & Male & MtLt & SCC & III & Right & $6 \times 6 \mathrm{~cm}$ & 4 months & 18 months alive Unknown \\
1997 Yoshizumi & 56 & Male MtLt & SCC & IV & Left & $1.5 \times 1.5 \mathrm{~cm}$ & 0 & 22 months alive + \\
1997 & Hata & 67 & Male & MtLt & SCC & III & Left & $6.5 \times 5.5 \mathrm{~cm}$ & 8 months & 14 months alive - \\
2004 & Nagano & 57 & Male MtLt & SCC & II & Left & $6.2 \times 4.8 \mathrm{~cm}$ & 3 months & unknown & Unknown \\
2006 & MM.Cho & 70 & Male LtAe & SCC & III & Left & $5 \times 4 \mathrm{~cm}$ & 8 months & 42 months alive + \\
2010 & Saito & 71 & Male & Mt & Adeno & III & Right & $2.5 \times 2 \mathrm{~cm}$ & 22 months & 71 months alive + \\
2013 & O'Sullivan KE & 50 & Male AeG & Adeno & II & Left & Unknown & 48 months & 48 months alive + \\
2016 & Our case & 83 & Male LtAe & SCC & III & Right & $2 \mathrm{~cm}$ & 14 months & 36 months alive -
\end{tabular}

SCC squamous cell carcinoma, Adeno adenocarcinoma, AM adrenal metastasis, EC esophageal carcinoma

despite the relatively small size. Metastasis from esophageal carcinoma was therefore suspected.

Adrenal metastasis in patients with esophageal carcinoma is very rare [12]. We found only eight cases between 1995 and 2015 (Table 1) [13-18]. The sex ratio showed a clear male predominance (male:female, 8:0). Median age at primary diagnosis was 63 years (range, 50-83 years). The adrenal metastasis was right-sided in three patients and left-sided in five. The median interval from the primary operation to discovery of metastasis was 8 months. Patients who underwent adrenalectomy survived for a median of 36 months (range, 14-71 months), with all achieving relatively long survival after adrenalectomy. Thus, in our very elderly patient, laparoscopic adrenalectomy was selected.

In general, chemotherapy is chosen for distant recurrence of esophageal carcinoma because of the oncological systemic status. Chemotherapy such as S-1 was considered for this patient. However, chemotherapy was not recommended for our patient because of his advanced age and concern about the side effects such as diarrhea. Thus, with sufficiently informed consent, surgical resection represents an option for solitary adrenal metastasis, even for those with distant recurrence. Best practice therapy including surgical resection and radiation therapy as well as chemotherapy is thus important to consider for patients with adrenal metastasis recurrence of esophageal carcinoma. In particular, surgical resection might be more important for solitary adrenal metastasis than for other distant metastases.

We report a case of prolonged recurrence-free survival (38 months) after laparoadrenalectomy for right adrenal metastasis following esophageal cancer surgery in a very elderly male. Surgical resection might be an option for solitary adrenal recurrence.

\section{Abbreviations}

CEA: Carcinoembryonic antigen; CT: Computed tomography; FDG: 8F-fluorodeoxyglucose; PET: Positron emission tomography; SCC: Squamous cell carcinoma; SUV: Standardized uptake value
Funding

The authors declare that this study was not funded externally.

\section{Authors' contributions}

NK performed the literature review and wrote the manuscript. YS, KN, KS, ST, and NM were involved in the clinical management of the patient. TO participated in literature review. TF, YS, and KN revised the manuscript. All authors were involved in the management of the patient. All authors read and approved the final manuscript.

Competing interests

The authors declare that they have no competing interests.

Consent for publication

The consent to publish is obtained from this patient.

\section{Publisher's Note}

Springer Nature remains neutral with regard to jurisdictional claims in published maps and institutional affiliations.

\section{Author details}

'Department of Gastroenterological Surgery, Okayama University Graduate School of Medicine, Dentistry and Pharmaceutical Sciences, 2-5-1

Shikata-cho, Kita-ku, Okayama 700-8558, Japan. ${ }^{2}$ Department of Surgery,

Shigei Medical Research Institute, Okayama, Japan.

Received: 2 February 2017 Accepted: 1 May 2017

Published online: 05 May 2017

References

1. Lerut T, De Leyn P, Coosemans W, Van Raemdonck D, Scheys I, LeSaffre E. Surgical strategies in esophageal carcinoma with emphasis on radical lymphadenectomy. Ann Surg. 1992;216:583-90.

2. Kato H. Classification of recurrent esophageal cancer after radical esophagectomy with two- or three-field lymphadenectomy. Anticancer Res. 2010;210:3461-8.

3. Abate E, DeMeester SR, Zehetner J, Oezcelik A, Ayazi S, Costales J, Banki F, Lipham JC, Hagen JA, DeMeester TR. Recurrence after esophagectomy for adenocarcinoma: defining optimal follow-up intervals and testing. J Am Coll Surg. 2010;210:428-35.

4. Nakamura T, Ota M, Narumiya K, Sato T, Ohki T, Yamamoto M, Mitsuhashi N. Multimodal treatment for lymph node recurrence of esophageal carcinoma after curative resection. Ann Surg Oncol. 2008;15:2451-7.

5. Kunisaki C, Makino H, Takagawa R, Yamamoto N, Nagano Y, Fujii S, Kosaka T, Ono HA, Otsuka Y, Akiyama H, Ichikawa Y, Shimada H. Surgical outcomes in esophageal cancer patients with tumor recurrence after curative esophagectomy. J Gastrointest Surg. 2008;12:802-10.

6. Huddy JR, Thomas RL, Worthington TR, Karanjia ND. Liver metastases from esophageal carcinoma: is there a role for surgical resection? Dis Esophagus. 2015;28:483-7. 
7. Chen F, Sato K, Sakai H, Miyahara R, Bando T, Okubo K, Hirata T, Date H. Pulmonary resection for metastasis from esophageal carcinoma. Interact Cardiovasc Thorac Surg. 2008;7:809-12.

8. Mariette C, Balon JM, Piessen G, Fabre S, Van Seuningen I, Triboulet JP. Pattern of recurrence following complete resection of esophageal carcinoma and factors predictive of recurrent disease. Cancer. 2003;97:1616-23.

9. Ichikawa H, Kosugi S, Kanda T, Ishikawa T, Yajima K, Akazawa K, Suzuki T, Wakai T. Prognostic significance of initial recurrence site in hematogenous recurrence of esophageal squamous cell carcinoma. Hepatogastroenterology. 2014;61:2241-6.

10. Boland GW, Blake MA, Holalkere NS, Hahn PF. PET/CT for the characterization of adrenal masses in patients with cancer: qualitative versus quantitative accuracy in 150 consecutive patients. AJR Am J Roentgenol. 2009;192:956-62.

11. Okada M, Shimono T, Komeya Y, Ando R, Kagawa Y, Katsube T, Kuwabara M, Yagyu Y, Kumano S, Imaoka I, Tsuchiya N, Ashikaga R, Hosono M, Murakami T. Adrenal masses: The value of additional fluorodeoxyglucose-positron emission tomography/computed tomography (FDG-PET/CT) in differentiating between benign and malignant lesions. Ann Nucl Med. 2009;23:349-54.

12. Young WF. Clinical practice. The incidentally discovered adrenal mass. N Engl J Med. 2007;356:601-10

13. Ninomiya I, Okamoto K, Tsukada T, Kinoshita J, Oyama K, Fushida S, Osugi H, Ohta T. Recurrence patterns and risk factors following thoracoscopic esophagectomy with radical lymph node dissection for thoracic esophageal squamous cell carcinoma. Mol Clin Oncol. 2016:4:278-84.

14. Hata S, Amano S, Sakurai K, Fujii H, Paku E, Kano H, Oida N, Miyake H, Fukuzawa M, Sugitani M. Resection of adrenal metastasis from esophageal cancer: a case report. Jpn J Gastroenterol Surg. 2000;33:721-4.

15. Saito H, Shuto K, Ota T, Toma T, Ohira G, Natsume T, Uesato M, Akutsu Y, Kono T, Matsubara H. A case of long-term survival after resection for postoperative solitary adrenal metastasis from esophageal adenocarcinoma. Gan to Kagaku Ryoho. 2010;37:2406-8.

16. Cho MM, Kobayashi K, Aoki T, Nishioka K, Yoshida K, Hatano N, Hirose H, Moon JH, Matsumoto T, Uemura Y, Kaneko T, Okagawa K. Surgical resection of solitary adrenal metastasis from esophageal carcinoma following esophagectomy. Dis Esophagus. 2007;20:79-81.

17. Yoshizumi Y, Morisaki Y, Koike H, Shibata H, Yanagawa R, Sugiura Y, Tanaka S. Successful combined resection of carcinoma of the esophagus and adrenal metastasis: report of a case. Surg Today. 1997;27:330-3.

18. Shimada Y, Imamura M, Kanda Y. Resection of adrenal metastasis from esophageal carcinoma after esophagectomy without recurrence for one and half year: a case report. Arch Jpn Chir. 1992;61:86.

\section{Submit your manuscript to a SpringerOpen ${ }^{\circ}$ journal and benefit from:}

- Convenient online submission

- Rigorous peer review

- Immediate publication on acceptance

- Open access: articles freely available online

- High visibility within the field

- Retaining the copyright to your article 